\title{
Harmonic functions on the lattice: Absolute monotonicity and propagation of smallness
}

\author{
Gabor Lippner and Dan Mangoubi
}

\begin{abstract}
In this work we establish a connection between two classical notions, unrelated so far: Harmonic functions on the one hand and absolutely monotonic functions on the other hand. We use this to prove convexity type and propagation of smallness results for harmonic functions on the lattice.
\end{abstract}

\section{Introduction}

The aim of the present paper is to discuss convexity properties of discrete harmonic functions. One of our main discoveries is that if $u$ is a harmonic function then $\Delta^{k} u^{2}$ is non-negative for every $k \in \mathbb{N}$, and that this fact can be used to prove convexity type results. A minor byproduct of this work is an elementary new proof of the well known Liouville property and finite dimensionality of harmonic functions of bounded polynomial growth in $\mathbb{Z}^{d}$. Some of our results can be adapted also to harmonic functions in $\mathbb{R}^{d}$, but we do not pursue this direction here, since working in the discrete world, we are faced with several challenges which do not exist in the continuous world.

\subsection{Background: Hadamard's Three Circles Theorem, Agmon's Theorem.}

The connection between holomorphic functions and convexity goes back to Hadamard. For $f$ holomorphic, let $M(r):=\max _{B(r)}|f|$. Hadamard proved the Three Circles Theorem: $M(2 r) \leq \sqrt{M(r) M(4 r)}$ or equivalently, $\log M(r)$ is a convex function as a function of $\log r$. Once this theorem is 
known, a version for harmonic function $u$ is naturally sought after. It is readily seen that $M_{u}(r):=\max _{B(r)}|u|$ is a convex function as a function of $\log r$. However, only approximately is it true that $\log M_{u}$ is a convex function of $\log r$. The approximate logarithmic convexity of $M_{u}(r)$ is due to Landis' school [Lan63, Ch. II.2]. Apparently, Agmon was the first to observe that if one replaces the function $M_{u}(r)$ with an $L^{2}$-version on a sphere then one gets a precise logarithmic convexity result:

Theorem 1.1 (Agm66]). Let $u$ be a harmonic function defined in the open ball of radius $R, B(0, R) \subset \mathbb{R}^{d}$. Let the $L^{2}$-growth function be defined by

$$
q_{u}(r):=\frac{1}{\left|S^{d-1}(r)\right|} \int_{S^{d-1}(r)} u^{2} d \sigma_{r} .
$$

Then $\log q_{u}$ is a convex function of $\log r$ for $\log r<\log R$.

Here we should also mention that the idea to consider the integral of $u^{2}$ on arcs (in two dimensions) is due to Carleman, and in particular, a second order differential inequality closely related to the logarithmic convexity was proved in Car33. The observation of Agmon was that in a specific setting Carleman's differential inequality can be strengthened to obtain the logarithmic convexity.

\subsection{Main result I: Discrete absolute monotonicity}

In fact, it turns out that a stronger property of $q_{u}(r)$ holds. We recall the following definition

Definition 1.2 ([Ber14] $)$. A $C^{\infty}$-function $f:[0, T) \rightarrow \mathbb{R}$ is called absolutely monotonic in $[0, T)$ if $f$ and all its derivatives are non-negative.

A fundamental property of absolutely monotonic functions is given by a theorem of S. Bernstein (see also [Wid41, Ch. IV]):

Theorem 1.3 ([Ber14]). An absolutely monotonic function in $[0, T)$ is realanalytic in $(0, T)$. Moreover, it extends to a real-analytic function in $(-T, T)$.

We observe

Theorem 1.4. If $u: B(0, R) \rightarrow \mathbb{R}$ is harmonic, then the function $q_{u}(r)$ is absolutely monotonic in $[0, R)$. 
Using Theorem 1.3, it is an exercise to check that absolute monotonicity implies logarithmic convexity on the scale of $\log r$ [PS72, part II, problem 123]. Theorem 1.4 is easily understood by considering the spherical harmonics expansion of harmonic functions.

A central idea we try to convey in this paper is that the strengthened Agmon's Theorem 1.4 lends itself more naturally to a discrete analogue than Theorem 1.1. To explain this, we let $\left(X_{n}\right)_{n=0}^{\infty}$ be a simple random walk on $\mathbb{Z}^{d}$ starting at 0 , and $\left(Y_{t}\right)_{t \geq 0}$ be a continuous time random walk on $\mathbb{Z}^{d}$ starting at 0 . We introduce the following discrete $L^{2}$-growth functions

Definition 1.5. Let $B_{R} \subseteq \mathbb{Z}^{d}$ be the closed ball of radius $R$ centered at 0 . The discrete growth function of $u: B_{R} \rightarrow \mathbb{R}$ is defined by

$$
\forall 0 \leq n \leq R, \quad Q_{u}(n):=\mathbb{E}\left(u\left(X_{n}\right)^{2}\right) .
$$

If $u$ is globally defined we set

$$
Q_{c, u}(t):=\mathbb{E}\left(u\left(Y_{t}\right)^{2}\right) .
$$

Remark 1.6. $Q_{c, u}(t)$ could be infinite. However, there exists $T \geq 0$ such that $Q_{c, u}(t)<\infty$ for $0<t<T$ and $Q_{c, u}(t)=\infty$ for $t>T$. Indeed, if we denote by $p_{c}(t, x)$ the continuous time heat kernel on $\mathbb{Z}^{d}$, then it follows directly from the heat equation and the fact that $p_{c} \geq 0$ that $e^{t} p_{c}(t, x)$ is non-decreasing in $t$. The claim follows since $Q_{c, u}(t, x)=e^{-t} \sum_{x \in \mathbb{Z}^{d}} u(x)^{2} e^{t} p_{c}(t, x)$.

Remark. Observe that unlike the $\mathbb{R}^{d}$ version (1), where we consider spheres in space, here we consider spheres in time.

We define

Definition 1.7. Let $f: \mathbb{N}_{0} \cap[0, R] \rightarrow \mathbb{R}$ be a discrete function. We say that $f$ is absolutely monotonic in $[0, R]$ in the discrete sense if $f^{(k)}(n) \geq 0$ for all $k, n \in \mathbb{N}_{0}$ such that $k+n \leq R$. Here $f^{(k)}$ is the $k$-th forward discrete derivative, i.e. $f^{\prime}(n)=f(n+1)-f(n)$ and $f^{(k)}:=f^{(k-1)^{\prime}}$.

Example. $f(n)=\left(\begin{array}{l}n \\ k\end{array}\right)$ is absolutely monotonic in $\mathbb{N}_{0}$ in the discrete sense.

A main result we prove is

Theorem 1.8. Let $u: B_{R} \rightarrow \mathbb{R}$ be harmonic. Then, the function $Q_{u}$ is absolutely monotonic in $[0, R]$ in the discrete sense. If $u$ is globally defined and $Q_{c, u}$ is finite on $[0, T)$, then it is absolutely monotonic in $[0, T)$. 
Besides the main applications of Theorem 1.8 discussed below, we also immediately obtain a new simple proof of the finite dimensionality of the space of harmonic functions of bounded polynomial growth.

Corollary 1.9. Let $u: \mathbb{Z}^{d} \rightarrow \mathbb{R}$ be a non-zero harmonic function. Suppose

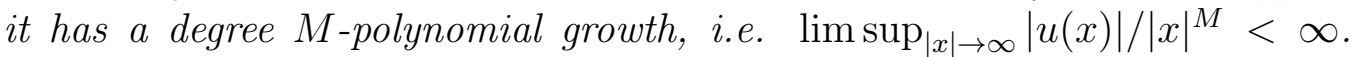
Then $u$ must be a polynomial of degree at most $M$. In particular, the space of degree $M$-polynomial growth harmonic functions is finite dimensional. Moreover, the function $u$ cannot vanish identically on a ball of radius $M$.

\subsection{Main result II: Discrete Three Circles Theorems}

Our main application of Theorem 1.8 gives two discrete analogues of Hadamrad's Three Circles Theorem.

It is easier to begin with the setting of a globally defined harmonic function and the continuous time random walk.

Theorem 1.10. Let $u: \mathbb{Z}^{d} \rightarrow \mathbb{R}$ be harmonic. Then

$$
Q_{c, u}(2 t) \leq \sqrt{Q_{c, u}(t) Q_{c, u}(4 t)}
$$

A version of Theorem 1.10 for the discrete time random walk turns out to be more difficult. The reason is that an error term must appear (for $d \geq 2$ ). Indeed, for any $R \in \mathbb{N}$ there exists a harmonic polynomial $u: \mathbb{Z}^{d} \rightarrow \mathbb{R}$ such that $\left.u\right|_{B_{2 R}}$ is not zero and $\left.u\right|_{B_{R}} \equiv 0$. Then, $Q_{u}$ cannot satisfy an inequality of the form $Q_{u}(2 n) \leq \sqrt{Q_{u}(n) Q_{u}(4 n)}$ for all $n \in \mathbb{N}$.

Theorem 1.11. Let $u: B_{4 R} \rightarrow \mathbb{R}$ be harmonic and let $0 \leq \varepsilon \leq 0.5$. Then

$$
Q_{u}(2 n) \leq \sqrt{e^{n^{-2 \varepsilon}} Q_{u}(n) Q_{u}(4 n)}+2^{-n^{0.5-\varepsilon}} Q_{u}(4 n)
$$

for all $16<n \leq R$.

Observe that the constant $e^{n^{-2 \varepsilon}}$ tends to 1 and the error term $2^{-n^{0.5-\varepsilon}}$ goes to 0 as $n$ goes to infinity if $0<\varepsilon<0.5$. Theorem 1.11 is inspired from [GM13, where an $L^{\infty}$ variant with essentially the same error term is proved.

In case we have an a priori bound on the growth of $u$ we show that the error term can be dropped for large $n$ : 
Theorem 1.12. Let $u: \mathbb{Z}^{d} \rightarrow \mathbb{R}$ be a harmonic polynomial of degree $M$. Let $0 \leq \varepsilon<0.5$. Then

$$
Q_{u}(2 n) \leq \sqrt{e^{n^{-2 \varepsilon}} Q_{u}(n) Q_{u}(4 n)}
$$

for all $n$ such that $n^{1-2 \varepsilon}>M^{2}$ and $n>16$.

It would be most interesting to understand the sharp error term in Theorem 1.11. If we do not fix the dimension of the lattice, the error term proved is optimal.

Theorem 1.13. For all $\varepsilon>0, n_{0} \in N, C>0$ and $d>d_{0}\left(\varepsilon, n_{0}, C\right)$ there exists a harmonic function $u: \mathbb{Z}^{d} \rightarrow \mathbb{R}$ such that

$$
Q_{u}(2 n)>C \sqrt{Q_{u}(n) Q_{u}(4 n)}+2^{-n^{0.5+\varepsilon}} Q_{u}(4 n)
$$

for some $n>n_{0}$.

However, we conjecture that the error term proved is optimal in every dimension.

Conjecture 1.14. For all $\varepsilon>0, n_{0} \in N, C>0$ and $d \geq 2$, there exists a harmonic function $u: \mathbb{Z}^{d} \rightarrow \mathbb{R}$ such that

$$
Q_{u}(2 n)>C \sqrt{Q_{u}(n) Q_{u}(4 n)}+2^{-n^{0.5+\varepsilon}} Q_{u}(4 n)
$$

for some $n>n_{0}$.

In Section 5 we discuss Conjecture 1.14.

Remark 1.15. It is interesting to point out the connection between the constant in front of the main term in the RHS of (21) and the exponent $-n^{\beta}$ in the error term in (21). We prove that if $\beta<0.5$ then the constant tends to 1 for large $n$, and we believe that $\beta$ cannot be made bigger than 0.5 even at the expense of an arbitrarily large constant. We do not know what to expect when $\beta=0.5$, besides what we prove in Theorem 1.11.

It is worth noting that if the ratios $1: 2: 4$ in Theorem 1.11 are perturbed, the error term drops dramatically, hinting at a delicate phase change phenomenon, which further motivates our interest in the optimal error term. 
Theorem 1.16. Let $u: B_{5 R} \rightarrow \mathbb{R}$ be harmonic. Let $0<\delta<1 / 4$. Then

$$
Q_{u}(2 n) \leq \sqrt{Q_{u}(n) Q_{u}(\lceil(4(1+\delta) n\rceil)}+2^{-2 n \delta} Q_{u}(\lceil(4(1+\delta) n\rceil)
$$

for all $0 \leq n \leq R$

However, for three concentric circles of any aspect ratio, if one adjusts the main term correspondingly the error term is stable.

Theorem 1.17. Let $1<P<p P$. Then, for any harmonic $u: B_{p P} \rightarrow \mathbb{R}$ and any $0 \leq \varepsilon \leq 0.5$

$$
Q_{u}(\lfloor P n\rfloor) \leq e^{c n^{-2 \varepsilon}} Q_{u}(n)^{\alpha} Q_{u}(\lceil p P n\rceil)^{1-\alpha}+p^{-n^{0.5-\varepsilon}} Q_{u}(\lceil p P n\rceil),
$$

for all $n_{0} \leq n \leq R$, where $n_{0}=n_{0}(p, P), P^{\alpha}=p^{1-\alpha}, c=2\left(\alpha P+(1-\alpha) \frac{1}{p}-1\right)$.

The proof of Theorem 1.17 is given for $\alpha=1 / 2$ (see Section 1.3, Theorem 1.11). The other cases are obtained by a slight modification and we omit the details.

Organization of the paper. In Section 2 we explain our crucial observation: Positivity of $\Delta^{k} u^{2}$ for a harmonic function $u$, and then we use it to prove the discrete analogue of the strengthened Agmon's theorem, Theorem 1.8, and Corollary 1.9, In Section 3 we prove the discrete versions of the Three Circles Theorem announced in Section 1.3. In Section 4 we show that the error term in Theorem 1.11 is optimal in a weak sense (Theorem 1.13). In Section 5 we discuss Conjecture 1.14.

Acknowledgments. Our preliminary results in the discrete setting involved the growth function at stopping times on spheres. We heartily thank Gady Kozma for his suggestion to try replacing the spheres in space by spheres in time, as this idea simplified and clarified most of our results. We are very grateful to Eugenia Malinnikova who explained to one of us her joint work GM13 which in turn inspired our statements of Theorem 1.11 and Conjecture 1.14. We also thank Eugenia for her valuable comments and helpful discussions. We owe our gratitude to Matthias Keller for helping us explore relevant directions. We thank Itai Benjamini, Bo'az Klartag and Gady Kozma for helpful discussions. We are very grateful to Shing-Tung Yau for his continuous support of both of us. We thank the anonymous 
referees for their valuable comments and for bringing to our attention the papers [JLS14] and Car33. G.L. gratefully acknowledges the support of AFOSR grant FA9550-09-1-0090. D.M. gratefully acknowledges the support of ISF grant no. 225/10. Both authors gratefully acknowledge the support of BSF grant no. 2010214.

\section{Laplacian powers of a harmonic function squared}

In this section we prove Theorem 1.8 and conclude Corollary 1.9. The heart of the matter in the proof of Theorem 1.8 is the following observation which we believe to be interesting in its own right.

Theorem 2.1. Let $u$ be a harmonic function on a Cayley graph of a finitely generated Abelian group or on $\mathbb{R}^{d}$. Then $\Delta^{k}\left(u^{2}\right)$ is non-negative for all $k \geq 0$.

We emphasize that in the proof of Theorem [1.8, the harmonicity of $u$ is used only through the application of Theorem 2.1

Proof of Theorem 2.1. The theorem follows by induction on $k$ from Claim 2.2 below.

Claim 2.2. Let $(A, S)$ be a finitely generated Abelian group with a finite set of generators $S$. Let $u: \operatorname{Cay}(A, S) \rightarrow \mathbb{R}$ or $u: \mathbb{R}^{d} \rightarrow \mathbb{R}$ be harmonic, then $\Delta\left(u^{2}\right)$ is a sum of squares of harmonic functions.

Proof of Claim 2.2. We give the proof for Cayley graphs. The proof in $\mathbb{R}^{d}$ is similar.

$$
\begin{aligned}
\left(\Delta u^{2}\right)(x) & =\frac{1}{|S|} \sum_{s \in S}\left(u(x+s)^{2}-u(x)^{2}\right) \\
& =\frac{1}{|S|} \sum_{s \in S}(u(x+s)-u(x))^{2}+2 u(x)(u(x+s)-u(x)) \\
& =\frac{1}{|S|} \sum_{s \in S}(u(x+s)-u(x))^{2}+2 u(x)(\Delta u)(x) \\
& =\frac{1}{|S|} \sum_{s \in S}(u(x+s)-u(x))^{2} .
\end{aligned}
$$


Finally, since translation commutes with the Laplacian on Abelian groups, $x \mapsto u(x+s)-u(x)$ is a harmonic function.

Remark 2.3. For later reference we record here a convenient related formula. For a generator $s \in S$ we set

$$
u_{s}(x):=u(x+s)-u(x)
$$

Iterating the proof of Claim 2.2 shows that for a harmonic function $u$

$$
\Delta^{k} u^{2}=\frac{1}{|S|^{k}} \sum_{s_{1}, s_{2}, \ldots, s_{k} \in S}\left(u_{s_{1} s_{2} \ldots s_{k}}\right)^{2} .
$$

\subsection{Proof of Theorem 1.8}

We prove absolute monotonicity of the growth function of a harmonic function. We begin with the following nice identity:

Lemma 2.4. Let $f: \mathbb{Z}^{d} \rightarrow[0, \infty)$ be any function. Let $E_{f}(n):=\mathbb{E} f\left(X_{n}\right)$. If $\mathbb{E}\left|f\left(Y_{t}\right)\right|<\infty$ let $E_{c, f}(t):=\mathbb{E} f\left(Y_{t}\right)$. Then

$$
E_{f}^{(k)}(n)=\mathbb{E}\left(\left(\Delta^{k} f\right)\left(X_{n}\right)\right),
$$

and

$$
E_{c, f}^{(k)}(t)=\mathbb{E}\left(\left(\Delta^{k} f\right)\left(Y_{t}\right)\right)
$$

Proof of Lemma 2.4. The Lemma follows from the case $k=1$. Let

$$
p(n, x)=\operatorname{Prob}\left(X_{n}=x\right) .
$$

The function $p$ satisfies the heat equation:

$$
p(n+1, x)-p(n, x)=(\Delta p)(n, x),
$$

where $\Delta p$ is the Laplacian with respect to the space parameter, $x$. Hence,

$$
\begin{aligned}
E_{f}^{\prime}(n)=E_{f}(n+1)-E_{f}(n)=\sum_{x} f(x)(p(n+1, x)-p(n, x)) \\
=\sum_{x} f(x)(\Delta p)(n, x)=\sum_{x}(\Delta f)(x) p(n, x)=\mathbb{E}\left((\Delta f)\left(X_{n}\right)\right),
\end{aligned}
$$


where we have used the self adjointness of the Laplace operator.

In the continuous case the line of proof is the same, but we have to treat convergence issues. First, we check that differentiation of the infinite sum can be done term by term. To that end, it is sufficient to check that there exists a $\delta>0$ such that for all $t \in[0, T)$

$$
\sum_{x} \sup _{\left|t^{\prime}-t\right|<\delta}\left|\partial_{t} p_{c}\left(t^{\prime}, x\right)\right||f(x)|<\infty
$$

We have

$$
\left|\partial_{t} p_{c}\left(t^{\prime}, x\right)\right|=\left|\Delta p_{c}\left(t^{\prime}, x\right)\right| \leq p_{c}\left(t^{\prime}, x\right)+(1 / 4) \sum_{y \sim x} p_{c}\left(t^{\prime}, y\right) .
$$

Observe that $p_{c}\left(t^{\prime}, v\right) \leq e^{\delta} p_{c}(t, v)$ for any $v \in \mathbb{Z}^{d}$ by Remark 1.6. Hence, it is enough to show that $\sum_{x} \sum_{y \sim x} p_{c}(t, y)|f(x)|<\infty$. However, for any $t$ and $y \sim x$ we have $p_{c}(t+\delta, x)=\sum_{z} p_{c}(t, z) p_{c}(\delta, x-z) \geq p_{c}\left(\delta, e_{1}\right) p_{c}(t, y)$. Then,

$$
\sum_{x} \sum_{y \sim x} p_{c}(t, y)|f(x)| \leq \frac{4}{p_{c}\left(\delta, e_{1}\right)} \sum_{x} p_{c}(t+\delta, x)|f(x)| \leq \frac{4 e^{\delta}}{p_{c}\left(\delta, e_{1}\right)} E_{c,|f|}(t)<\infty
$$

as desired. Second, we need to justify the reordering of the terms that is used to prove the identity $\sum_{x} f(x)\left(\Delta p_{c}\right)(t, x)=\sum_{x}(\Delta f)(x) p_{c}(t, x)$. For this, it is enough to check that

$$
\forall 1 \leq j \leq 2 d, \quad \sum_{x} p_{c}\left(t, x+e_{j}\right)|f(x)|<\infty .
$$

Again, we apply the same argument as before:

$$
p_{c}\left(t, x+e_{j}\right) \leq \frac{e^{\delta} p_{c}(t, x)}{p_{c}\left(\delta, e_{1}\right)} .
$$

We have all the ingredients now to present

Proof of Theorem 1.8. By Lemma 2.4

$$
Q_{u}^{(k)}(n)=\mathbb{E}\left(\left(\Delta^{k} u^{2}\right)\left(X_{n}\right)\right) .
$$

By Theorem 2.1 the expression on the RHS is non-negative. The proof for $Q_{c, u}$ is similar. 


\subsection{The Newton series of the growth functions}

In this section we prove the following useful formulas:

Theorem 2.5. Let $u: B_{R} \rightarrow \mathbb{R}$ be an arbitrary function. Then

$$
\forall 0 \leq n \leq R, \quad Q_{u}(n)=\sum_{k=0}^{R}\left(\Delta^{k} u^{2}\right)(0)\left(\begin{array}{l}
n \\
k
\end{array}\right)
$$

In addition, if $u$ is globally defined and $Q_{c, u}(t)<\infty$ then

$$
Q_{c, u}(t)=\sum_{k=0}^{\infty}\left(\Delta^{k} u^{2}\right)(0) \frac{t^{k}}{k !}
$$

The proof of Theorem 2.5 is based on Lemma 2.4, Theorem 1.3 and the following classical theorem on finite differences.

Let $F: \mathbb{N}_{0} \rightarrow \mathbb{R}$ be a discrete function. Let $F^{(k)}$ be as in Definition 1.7.

Theorem 2.6 (Newton series). The function $F: \mathbb{N}_{0} \rightarrow \mathbb{R}$ can be uniquely written in the form

$$
F(n)=\sum_{k=0}^{\infty} a_{k}\left(\begin{array}{l}
n \\
k
\end{array}\right)
$$

Moreover, $a_{k}=F^{(k)}(0)$.

Although standard, we reproduce the short proof for completeness.

Proof of Theorem [2.6. We can write

$$
F^{(k)}(n)=\sum_{j=0}^{k}(-1)^{k+j}\left(\begin{array}{l}
k \\
j
\end{array}\right) F(n+j) .
$$

Hence

$$
\begin{aligned}
\sum_{k=0}^{\infty} F^{(k)}(0)\left(\begin{array}{l}
n \\
k
\end{array}\right) & =\sum_{k=0}^{n} \sum_{j=0}^{k}(-1)^{k+j}\left(\begin{array}{l}
k \\
j
\end{array}\right)\left(\begin{array}{l}
n \\
k
\end{array}\right) F(j) \\
& =\sum_{j=0}^{n}(-1)^{j} F(j) \sum_{k=j}^{n}(-1)^{k}\left(\begin{array}{l}
k \\
j
\end{array}\right)\left(\begin{array}{l}
n \\
k
\end{array}\right)=
\end{aligned}
$$




$$
\begin{gathered}
=\sum_{j=0}^{n}(-1)^{j} F(j) \sum_{l=0}^{n-j}(-1)^{l+j}\left(\begin{array}{c}
l+j \\
j
\end{array}\right)\left(\begin{array}{c}
n \\
l+j
\end{array}\right) \\
=\sum_{j=0}^{n} F(j)\left(\begin{array}{c}
n \\
j
\end{array}\right) \sum_{l=0}^{n-j}(-1)^{l}\left(\begin{array}{c}
n-j \\
l
\end{array}\right)=\sum_{j=0}^{n} F(j)\left(\begin{array}{c}
n \\
j
\end{array}\right) \delta_{n-j, 0}=F(n) .
\end{gathered}
$$

Proof of Theorem 2.5. Let $f: \mathbb{Z}^{d} \rightarrow \mathbb{R}$ be any function. By Lemma 2.4 and Theorem 2.6 we get

$$
E_{f}(n)=\sum_{k=0}^{n} E_{\Delta^{k} f}(0)\left(\begin{array}{l}
n \\
k
\end{array}\right) .
$$

It only remains to observe that $E_{\Delta^{k} f}(0)=\left(\Delta^{k} f\right)(0)$. If we take $f=u^{2}$ in $B_{R}$ and $f=0$ outside $B_{R}$ we get the first part of the theorem.

We move to the second part. By Theorem 1.8 and Theorem 1.3 we know that

$$
Q_{c, u}(t)=\sum_{k=0}^{\infty} Q_{c, u}^{(k)}(0) \frac{t^{k}}{k !} .
$$

The second part of the theorem now follows from formula (5) and Lemma 2.4.

\subsection{Proof of Corollary 1.9}

In this section we show that Theorem 1.8 immediately implies that harmonic functions of polynomial growth are polynomials. At the same time it gives a quantitative estimate on the dimension of the space harmonic polynomials of degree at most $M$. This gives a simple proof of a well known result.

Proof of Corollary 1.9. By the assumption there exist $C, D>0$ such that

$$
\forall x \in \mathbb{Z}^{d}, \quad|u(x)|<C|x|^{M}+D
$$

We estimate $Q_{u}$ :

$$
Q_{u}(n)=\sum_{x} u(x)^{2} p(n, x)=\sum_{|x| \leq n} u(x)^{2} p(n, x) \leq\left(C n^{M}+D\right)^{2} .
$$


On the other hand, by Theorem 1.8 and Theorem 2.5 we know that

$$
\forall k, n \in \mathbb{N}_{0}, \quad Q_{u}(n) \geq\left(\Delta^{k} u^{2}\right)(0)\left(\begin{array}{l}
n \\
k
\end{array}\right) .
$$

Inequalities (6) and (7) imply

$$
\forall k>2 M, \quad \Delta^{k}\left(u^{2}\right)(0)=0 .
$$

To see that $u$ is a polynomial, observe that (8) and Remark 2.3 imply that

$$
\forall k>2 M \forall s_{1}, \ldots, s_{k} \in S, \quad u_{s_{1} s_{2} \ldots s_{k}}(0)=0
$$

and hence

$$
\forall s_{1}, \ldots, s_{2 M+1} \in S, \quad u_{s_{1} s_{2} \ldots s_{2 M+1}} \equiv 0 .
$$

i.e. $u$ is a polynomial of degree at most $2 M$. But of course, due to the growth assumption on $u, u$ is a polynomial of degree at most $M$.

Now, assume $u$ vanishes on the ball $B_{M}$. Then,

$$
\forall 0 \leq k \leq M, \quad \Delta^{k}\left(u^{2}\right)(0)=0 .
$$

In addition, by Remark 2.3

$$
\forall k>M, \quad \Delta^{k}\left(u^{2}\right)(0)=0 .
$$

We conclude from Theorem 2.5, (9) and (10) that

$$
\forall n \in \mathbb{N}_{0}, \quad Q_{u}(n)=0,
$$

which immediately implies $u \equiv 0$.

\section{Proof of Theorems $1.10-1.12$, and 1.16}

In this section we deduce from the absolute monotonicity of the discrete Agmon function (Theorem 1.8) logarithmic convexity type results.

For convenience we define

Definition 3.1. A function $f:(0, \infty) \rightarrow(0, \infty)$ is log-convex on a logarithmic scale (LCLS) if $t \mapsto \log f\left(e^{t}\right)$ is a convex function of $t \in \mathbb{R}$. If $f$ is continuous then $f$ is LCLS if $f(2 r) \leq \sqrt{f(r) f(4 r)}$ for all $r>0$. 
We begin with

Proof of Theorem 1.10. By Theorems 1.3 and 2.5

$$
Q_{c, u}(t)=\sum_{k=0}^{\infty} a_{k} t^{k}
$$

where all $a_{k} \geq 0$. Clearly, $a_{k} t^{k}$ is LCLS. It is well known that a sum and a limit of log-convex functions is log-convex (see Lemma 3.2 below).

We now move to the proof of Theorem 1.11. To explain also the case $\alpha=1 / 2$ of Theorem 1.17 we prove a slightly more general version:

Theorem [1.11. Let $P>1$. Let $u: B_{P^{2} R} \rightarrow \mathbb{R}$ be harmonic and let $0 \leq \varepsilon \leq 0.5$. Then

$$
Q_{u}(\lfloor P n\rfloor) \leq \sqrt{e^{n^{-2 \varepsilon}} Q_{u}(n) Q_{u}\left(\left\lceil P^{2} n\right\rceil\right)}+P^{-n^{0.5-\varepsilon}} Q_{u}\left(\left\lceil P^{2} n\right\rceil\right)
$$

for all $4 P^{2} \leq n \leq R$.

Proof of Theorem 1.11'. By Theorems 2.1 and 2.5 for $n \leq P R$ we know that

$$
Q_{u}(n)=\sum_{k=0}^{\infty} a_{k}\left(\begin{array}{l}
n \\
k
\end{array}\right)=\sum_{k=0}^{P R} a_{k}\left(\begin{array}{l}
n \\
k
\end{array}\right)
$$

where $a_{k} \geq 0$. We observe that inequality (11) is additive (see Lemma 3.2). Hence, it suffices to show

$$
\left(\begin{array}{c}
\lfloor P n\rfloor \\
k
\end{array}\right) \leq \sqrt{e^{n^{-2 \varepsilon}}\left(\begin{array}{l}
n \\
k
\end{array}\right)\left(\begin{array}{c}
\left\lceil P^{2} n\right\rceil \\
k
\end{array}\right)}+P^{-n^{0.5-\varepsilon}}\left(\begin{array}{c}
\left\lceil P^{2} n\right\rceil \\
k
\end{array}\right)
$$

for all $k, n \in \mathbb{N}_{0}$. It can be easily checked that (12) is satisfied for $k=0,1$ and all $n$. From this point on, we assume $k \geq 2$.

Suppose that $n^{1-2 \varepsilon} \geq k^{2}$ :

$$
\begin{aligned}
& \frac{\left(\begin{array}{c}
\lfloor P n\rfloor \\
k
\end{array}\right)^{2}}{\left(\begin{array}{c}
n \\
k
\end{array}\right)\left(\begin{array}{c}
\left\lceil P^{2} n\right\rceil \\
k
\end{array}\right)} \leq \frac{\prod_{j=0}^{k-1}(P n-j)^{2}}{\prod_{j=0}^{k-1}(n-j)\left(P^{2} n-j\right)}=\prod_{j=0}^{k-1} \frac{P^{2} n^{2}-2 P n j+j^{2}}{P^{2} n^{2}-\left(P^{2}+1\right) n j+j^{2}} \\
& =\prod_{j=0}^{k-1}\left(1+\frac{(P-1)^{2} n j}{P^{2} n^{2}-\left(P^{2}+1\right) n j+j^{2}}\right) \leq \prod_{j=0}^{k-1}\left(1+\frac{(P-1)^{2} j}{P^{2} n-\left(P^{2}+1\right) k}\right)
\end{aligned}
$$




$$
\stackrel{(*)}{\leq} \prod_{j=0}^{k-1}\left(1+\frac{j}{n}\right) \leq\left(1+\frac{k}{n}\right)^{k}=\left(1+\frac{1}{n / k}\right)^{\frac{n}{k} \frac{k^{2}}{n}} \leq e^{\frac{k^{2}}{n}} \leq e^{n^{-2 \varepsilon}}
$$

where in $(*)$ we used $P^{2} n-\left(P^{2}+1\right) k>(P-1)^{2} n$ which holds as long as $n \geq k^{2}$ and $n \geq 4 P^{2}$.

On the other hand, if $n^{1-2 \varepsilon} \leq k^{2}$ then

$$
\frac{\left(\begin{array}{c}
\lfloor P n\rfloor \\
k
\end{array}\right)}{\left(\begin{array}{c}
\left\lceil P^{2} n\right\rceil \\
k
\end{array}\right)} \leq \prod_{j=0}^{k-1} \frac{P n-j}{P^{2} n-j} \leq P^{-k} \leq P^{-n^{0.5-\varepsilon}} .
$$

Thus, we have in fact proved a slightly stronger inequality than (12), namely

$$
\left(\begin{array}{c}
\lfloor P n\rfloor \\
k
\end{array}\right) \leq \max \left\{\sqrt{e^{n^{-2 \varepsilon}}\left(\begin{array}{l}
n \\
k
\end{array}\right)\left(\begin{array}{c}
\left\lceil P^{2} n\right\rceil \\
k
\end{array}\right)}, P^{-n^{0.5-\varepsilon}}\left(\begin{array}{c}
\left\lceil P^{2} n\right\rceil \\
k
\end{array}\right)\right\}
$$

Proof of Theorem 1.12. By Theorem 2.5 we can write

$$
\forall n \in \mathbb{N}_{0}, \quad Q_{u}(n)=\sum_{k=0}^{M} \Delta^{k}\left(u^{2}\right)(0)\left(\begin{array}{l}
n \\
k
\end{array}\right) .
$$

Indeed, Remark 2.3 shows that if $k>m$ then $\Delta^{k}\left(u^{2}\right)(0)=0$. Since $n^{1-2 \varepsilon} \geq$ $M^{2}$ and $n>16$, for any $0 \leq k \leq M$ inequality (13) with $P=2$ applies and yields

$$
\left(\begin{array}{c}
2 n \\
k
\end{array}\right) \leq \sqrt{e^{n^{-2 \varepsilon}}\left(\begin{array}{c}
n \\
k
\end{array}\right)\left(\begin{array}{c}
4 n \\
k
\end{array}\right)}
$$

Theorem 2.1 and Lemma 3.2 complete the proof.

Proof of Theorem 1.16. While the idea is similar to the idea of the proof of Theorem 1.11 the estimates here are simpler.

Let $n \geq(1+1 /(4 \delta)) k$. Then

$$
\frac{\left(\begin{array}{c}
2 n \\
k
\end{array}\right)^{2}}{\left(\begin{array}{c}
n \\
k
\end{array}\right)\left(\begin{array}{c}
\lceil 4(1+\delta) n\rceil \\
k
\end{array}\right)} \leq \prod_{j=0}^{k-1} \frac{(2 n-j)^{2}}{(n-j)(4(1+\delta) n-j)} \leq 1
$$

where the last estimate is true since $n \geq(1+1 /(4 \delta)) k$. 
On the other hand, if $n \leq(1+1 /(4 \delta)) k$ then

$$
\frac{\left(\begin{array}{c}
2 n \\
k
\end{array}\right)}{\left(\begin{array}{c}
\lceil 4(1+\delta) n\rceil \\
k
\end{array}\right)}=\prod_{j=0}^{k-1} \frac{2 n-j}{4(1+\delta) n-j} \leq 2^{-k} \leq 2^{-4 \delta n /(4 \delta+1)} .
$$

To summarize

$$
\left(\begin{array}{c}
2 n \\
k
\end{array}\right) \leq \max \left\{\sqrt{\left(\begin{array}{l}
n \\
k
\end{array}\right)\left(\begin{array}{c}
\lceil 4(1+\delta) n\rceil \\
k
\end{array}\right)}, 2^{-4 \delta n /(4 \delta+1)}\left(\begin{array}{c}
\lceil 4(1+\delta) n\rceil \\
k
\end{array}\right)\right\} .
$$

The claim now follows as in the proof of Theorem 1.11 .

It remains to prove

Lemma 3.2. Let $f_{1}, f_{2}: \mathbb{N}_{0} \rightarrow \mathbb{R}^{+}$be functions such that

$$
f_{i}\left(n_{2}\right) \leq C_{1} \sqrt{f_{i}\left(n_{1}\right) f_{i}\left(n_{3}\right)}+C_{2} E\left(n_{1}\right) f_{i}\left(n_{3}\right)
$$

for some $n_{1}, n_{2}, n_{3} \in \mathbb{N}_{0}$, some constants $C_{1}, C_{2}>0$ and some function $E: \mathbb{N}_{0} \rightarrow \mathbb{R}^{+}$. Then the same holds for $f=f_{1}+f_{2}$.

Proof. The Lemma follows if we can show

$$
\sqrt{a b}+\sqrt{c d} \leq \sqrt{a+c} \cdot \sqrt{b+d}
$$

for all $a, b, c, d \geq 0$. This is true due to the Cauchy-Schwarz inequality.

\section{Proof of Theorem 1.13}

Let us now give examples of harmonic functions which exhibit the optimality of the error term in Theorem 1.11 if we do not fix the dimension of the lattice. The idea is to construct a function $u$ for which $Q_{u}(n)=\left(\begin{array}{l}n \\ k\end{array}\right)$ for any given $k$, and to analyze the respective error in a three circles theorem.

Proof of Theorem 1.13. Let $k \leq d$. Define $u_{k}: \mathbb{Z}^{d} \rightarrow \mathbb{R}$ by $u_{k}\left(x_{1}, \ldots, x_{d}\right)=$ $x_{1} x_{2} \cdots x_{k}$. It is easy to check that $u_{k}$ is harmonic when we take the generating set, $S$, of $\mathbb{Z}^{d}$ to be the standard one, i.e., we consider $\mathbb{Z}^{d}$ as the free abelian group generated by $S_{0}=\left\{e_{1}, \ldots, e_{d}\right\}$ and we take $S=S_{0} \cup-S_{0}$. By Theorem 2.5 and Remark 2.3 we see that

$$
Q_{u_{k}}(n)=\sum_{l=0}^{k} \frac{1}{|S|^{l}} \sum_{s_{1}, s_{2}, \ldots, s_{l} \in S}\left(u_{s_{1} s_{2} \ldots s_{l}}\right)^{2}(0)\left(\begin{array}{l}
n \\
l
\end{array}\right) .
$$


However $u_{s_{1} s_{2} \ldots s_{l}}(0)=0$ unless $l=k$ and the $s_{i}$ 's generate $\oplus_{j=1}^{k} \mathbb{Z} e_{j}$. In that case $u_{s_{1} s_{2} \ldots s_{k}}(0)^{2}=1$. Thus

$$
Q_{u_{k}}(n)=c_{d, k}\left(\begin{array}{l}
n \\
k
\end{array}\right)
$$

where $c_{d, k}=k ! / d^{k}$. The theorem now follows from Proposition 4.2 below.

Remark 4.1. We can in fact see from the previous proof that if $P$ is any polynomial of degree at most $d$ that is absolutely monotonic in the discrete sense, then there exists a harmonic polynomial $u: \mathbb{Z}^{d} \rightarrow \mathbb{R}$ such that $Q_{u}=P$.

Proposition 4.2. Let $C>0, \varepsilon>0$. Let $k>k_{0}(C, \varepsilon)$, and $n=k^{2} / \log k$. Then,

$$
\left(\begin{array}{c}
2 n \\
k
\end{array}\right)>C \sqrt{\left(\begin{array}{c}
n \\
k
\end{array}\right)\left(\begin{array}{c}
4 n \\
k
\end{array}\right)}+2^{-n^{0.5+\varepsilon}}\left(\begin{array}{c}
4 n \\
k
\end{array}\right) .
$$

Proof of Proposition 4.2.

$$
\begin{aligned}
\frac{\left(\begin{array}{c}
2 n \\
k
\end{array}\right)}{\left(\begin{array}{c}
4 n \\
k
\end{array}\right)} & =\prod_{j=0}^{k-1} \frac{2 n-j}{4 n-j}=\frac{1}{2^{k}} \prod_{j=0}^{k-1}\left(1-\frac{j}{4 n-j}\right) \\
& >\frac{1}{2^{k}}\left(1-\frac{k}{4 n-k}\right)^{k} \sim 2^{-k} k^{-1 / 4} \gg 2^{-n^{0.5+\varepsilon}}
\end{aligned}
$$

where the last estimate is true since $k \ll n^{0.5+\varepsilon / 2}$.

On the other hand since $k \gg n^{0.5}$ we have,

$$
\begin{aligned}
\frac{\left(\begin{array}{c}
2 n \\
k
\end{array}\right)^{2}}{\left(\begin{array}{c}
n \\
k
\end{array}\right)\left(\begin{array}{c}
4 n \\
k
\end{array}\right)}=\prod_{j=0}^{k-1} \frac{(2 n-j)^{2}}{(n-j)(4 n-j)}=\prod_{j=0}^{k-1}\left(1+\frac{j n}{(n-j)(4 n-j)}\right) \geq \\
\geq \prod_{j=0}^{k-1}\left(1+\frac{j}{4 n}\right) \geq\left(1+\frac{k}{8 n}\right)^{k / 2} \sim e^{k^{2} /(16 n)}=k^{1 / 16} \gg 1 .
\end{aligned}
$$




\section{Conjecture 1.14: A discussion}

We explain our intuition and motivation in Conjecture 1.14. First observe that the proof of Theorem 1.13 shows that if we could construct for a fixed dimension $d$ and any $k \in \mathbb{N}$ a harmonic function $u$ such that $Q_{u}(n)=\left(\begin{array}{l}n \\ k\end{array}\right)$ then the conjecture would follow by Proposition 4.2. However it seems that this is only possible to do for $k \leq d$ (see Remark 4.1). A natural way to try to construct a polynomial $u$ for which $Q_{u}$ would approximate $\left(\begin{array}{l}n \\ k\end{array}\right)$ is to start with the polynomial $u_{k}=\Re z^{k}$ for which $q_{u_{k}}(r)=C r^{2 k}$ and to discretize it. Here we recall an algorithm for the discretization process due to JerisonLevine-Sheffield [JLS14], the origins of which can be found in [Lov04].

\subsection{The Jerison-Levine-Sheffield construction}

We essentially describe the construction from [JLS14].

Notation. Given a sequence of functions $\left(F_{k}\right)_{k=0}^{\infty}: \mathbb{Z} \rightarrow \mathbb{R}$, and a multi-index $\alpha \in \mathbb{N}_{0}^{d}$, we define the function $F_{\alpha}: \mathbb{Z}^{d} \rightarrow \mathbb{R}$ by

$$
\forall x=\left(x_{1}, \ldots, x_{d}\right) \quad F_{\alpha}(x):=\prod_{l=1}^{d} F_{\alpha_{l}}\left(x_{l}\right) .
$$

Theorem 5.1 (Correspondence principle). Let $F_{k}: \mathbb{Z} \rightarrow \mathbb{R}, k \in \mathbb{N}_{0}$, be a sequence of functions such that $\Delta F_{1}=\Delta F_{0}=0$, and $\Delta F_{k}=A F_{k-2}$ for all $k \geq 2$ and for some $A \in \mathbb{R}$. Let

$$
P(x)=\sum_{|\alpha| \leq M} a_{\alpha} \frac{x^{\alpha}}{\alpha !}
$$

be a harmonic polynomial in $\mathbb{R}^{d}$. Then, the polynomial

$$
P^{\mathbb{Z}}(x)=\sum_{|\alpha| \leq M} a_{\alpha} F_{\alpha}(x),
$$

is harmonic in $\mathbb{Z}^{d}$.

The proposition below gives a concrete sequence of functions $F_{k}: \mathbb{Z}^{d} \rightarrow \mathbb{R}$ which can be plugged into the correspondence principle. 
Proposition 5.2. Let $F_{k}: \mathbb{Z} \rightarrow \mathbb{R}, k \in \mathbb{N}_{0}$, be defined as follows:

$$
\begin{aligned}
F_{0}(x) & =1, \\
\forall k>0 \quad F_{k}(x) & =\left(\begin{array}{c}
x+\frac{k-1}{2} \\
k
\end{array}\right)=\frac{1}{k !} \prod_{j=0}^{k-1}\left(x+\frac{k-1}{2}-j\right) .
\end{aligned}
$$

Then

(a) $F_{k}^{\prime}(x)=F_{k-1}\left(x+\frac{1}{2}\right)$

(b) $\Delta F_{1}=\Delta F_{0}=0$ and $\Delta F_{k}=\frac{1}{2} F_{k-2}$ for all $k \geq 2$.

Proof. First we check that part(b) follows from part (a). In fact, by part (a) $F_{k}^{\prime \prime}(x)=F_{k-1}^{\prime}\left(x+\frac{1}{2}\right)=F_{k-2}(x+1)$. It remains to observe that $\Delta F(x)=$ $\frac{1}{2} F^{\prime \prime}(x-1)$. To prove part (a) we observe that

$$
\begin{aligned}
F_{k}(x+1) & =\frac{1}{k}\left(x+\frac{k+1}{2}\right) F_{k-1}\left(x+\frac{1}{2}\right) \\
\text { and } \quad F_{k}(x) & =\frac{1}{k}\left(x-\frac{k-1}{2}\right) F_{k-1}\left(x+\frac{1}{2}\right) .
\end{aligned}
$$

Remark. The family $F_{k}(x)=\left(\begin{array}{c}x+\underset{k}{\lfloor 2\rfloor}) \\ k\end{array}\right.$ is implicitly used in Lov04.

\subsection{Specialization of Conjecture 1.14}

Let $F_{k}: \mathbb{Z} \rightarrow \mathbb{R}$ be as in Proposition [5.2, Define

$$
\begin{aligned}
\forall k \geq 0 \quad S_{k}(x, y) & :=\sum_{j=0}^{\lfloor k / 2\rfloor}(-1)^{j} F_{k-2 j}(x) F_{2 j}(y), \\
\text { and } \forall k \geq 1 \quad T_{k}(x, y) & :=\sum_{j=0}^{\lfloor(k-1) / 2\rfloor}(-1)^{j} F_{k-(2 j+1)}(x) F_{2 j+1}(y) .
\end{aligned}
$$

Then, $S_{k}, T_{k}$ are harmonic in $\mathbb{Z}^{2}$. This can be immediately seen from the fact that $\Re(x+i y)^{k}$ and $\Im(x+i y)^{k}$ are harmonic in $\mathbb{R}^{2}$ and Theorem 5.1.

We believe that $S_{k}$ is a family of harmonic functions which gives the optimal error term in Theorem 1.11, namely, 
Conjecture 5.3. Let $C>0$. Then

$$
Q_{S_{k}}(2 n)>C \sqrt{Q_{S_{k}}(n) Q_{S_{k}}(4 n)}+2^{-n^{0.5+\varepsilon}} Q_{S_{k}}(4 n)
$$

for $k$ large enough and $n \sim k^{2} / \log k$.

\section{References}

[Agm66] S. Agmon, Unicité et convexité dans les problèmes différentiels, Séminaire de Mathématiques Supérieures, No. 13 (Été, 1965), Les Presses de l'Université de Montréal, Montreal, Que., 1966.

[Ber14] S. Bernstein, Sur la définition et les propriétés des fonctions analytiques d'une variable réelle, Math. Ann. 75 (1914), no. 4, 449-468 (French).

[Car33] T. Carleman, Sur une inégalité différentielle dans la thèorie des fonctions analytiques, C. R. Acad. Sci. Paris 196 (1933), 995-997 (French).

[GM13] M. Guadie and E. Malinnikova, A note on unique continuation for discrete harmonic functions (2013), preprint, available at arXiv:math/1306.1418,

[JLS14] D. Jerison, L. Levine, and S. Sheffield, Internal DLA and the Gaussian free field, Duke Math. J. 163 (2014), no. 2, 267-308.

[Lan63] E. M. Landis, Some questions in the qualitative theory of second-order elliptic equations (case of several independent variables), Uspehi Mat. Nauk 18 (1963), no. 1 (109), 3-62; English transl., Russian Math. Surveys 18 (1963), 1-62.

[Lov04] L. Lovász, Discrete analytic functions: an exposition, Surveys in differential geometry. Vol. IX, Surv. Differ. Geom., IX, Int. Press, Somerville, MA, 2004, pp. 241-273.

[PS72] G. Pólya and G. Szegö, Problems and theorems in analysis. Vol. I: Series, integral calculus, theory of functions, Springer-Verlag, New York, 1972. Translated from the German by D. Aeppli; Die Grundlehren der mathematischen Wissenschaften, Band 193.

[Wid41] D. V. Widder, The Laplace Transform, Princeton Mathematical Series, v. 6, Princeton University Press, Princeton, N. J., 1941. 
Gabor Lippner,

Department of Mathematics, Harvard University,

Cambridge, MA, USA

lippner@math . harvard . edu

Dan Mangoubi,

Einstein Institute of Mathematics, Hebrew University, Givat Ram, Jerusalem, Israel

mangoubi@math.huji.ac.il 